\title{
Regulation of innate and adaptive antitumor immunity by IAP antagonists
}

\author{
Stephanie K Dougan*,1,2 \& Michael Dougan**,2,3 \\ ${ }^{1}$ Department of Cancer Immunology \& Virology, Dana-Farber Cancer Institute, Boston, MA 02215, USA \\ ${ }^{2}$ Harvard Medical School, Boston, MA 02115, USA \\ ${ }^{3}$ Division of Gastroenterology, Department of Medicine, Massachusetts General Hospital, Boston, MA 02114, USA \\ *Author for correspondence: stephanie_dougan@dfci.harvard.edu \\ **Author for correspondence: mldougan@partners.org
}

Inhibition of the T-cell co-inhibitory checkpoint receptors or their ligands CTLA-4, PD-1 and PD-L1 using monoclonal antibodies has proven to be highly effective against many cancers. Yet many cancers remain resistant to checkpoint blockade, and durable remissions occur in only a minority of patients. Novel approaches to enhancing antitumor responses are thus necessary in order to expand the reach of these treatments. The inhibitor of apoptosis (IAP) protein family comprises a diverse group of proteins, many of which have immunoregulatory roles. Small molecule IAP antagonists have been developed and are undergoing early phase clinical testing. These drugs were initially developed to promote tumor cell apoptosis; however, a considerable body of work now indicates that IAP antagonists induce antitumor activity through modulation of innate and adaptive immunity. Primarily through inhibition of cellular (c)-IAP1 and c-IAP2, IAP antagonists can activate alternative NF-KB signaling, promoting B-cell survival, activation of dendritic cells and delivering a broad co-stimulatory signal to T cells. At the same time, IAP antagonists can promote tumor cell intrinsic sensitization to innate immune signals, and enhance tumor cell killing by inflammatory cytokines and phagocytic macrophages. These drugs thus represent an attractive investigational approach to immunotherapy, providing a positive signaling counterpart to the relief of signal inhibition conferred by checkpoint blockade.

First draft submitted: 11 December 2017; Accepted for publication: 14 March 2018; Published online: 29 May 2018

Keywords: C-IAP1/C-IAP2 $\bullet$ IAP antagonists $\bullet$ inhibitor of apoptosis proteins $\bullet$ SMAC mimetics $\bullet$ tumor immunotherapy

Inhibition of T-cell co-inhibitory 'checkpoint' receptors using monoclonal antibodies has proven to be highly effective clinically against a diverse range of malignancies [1-11]. Antibodies to CTLA-4, PD-1 and PD-L1 are now widely used in patients, and have been distinguished not only by their response rates, but more importantly, by the large number of durable remissions when compared with chemotherapy and oncogene-targeted therapies [1-11]. Many human tumors remain resistant to checkpoint blockade; however, novel classes of immune modulating drugs will be necessary to expand the reach of immunotherapy [2]. T cells appear to play the dominant role in response to checkpoint blockade, where effector cells that have been previously activated are often inhibited within the tumor microenvironment by high expression of co-inhibitory receptors [2,8]. Many of these T cells recognize neoantigens, mutated tumor proteins that generate novel nonself peptides displayed on MHC class I and MHC class II [12-14]. Reactivation of these cells by checkpoint blockade can induce a robust antitumor response, although tumors can acquire resistance to T-cell mediated killing by losing their ability to respond to T-cell-produced IFN $\gamma$, or by interfering with antigen processing and presentation [15-18].

Many novel strategies aim to deliver activating signals into the tumor microenvironment through the use of immune adjuvants $[2,19]$. Direct injection of TLR ligands, tumoricidal viruses and STING agonists are all under investigation as therapies that could activate intratumoral antigen-presenting cells (APC)s $[2,19]$. APC activation leads to increased expression of co-stimulatory ligands, providing $\mathrm{T}$ cells with a positive signal to couple with the blockade of co-inhibition provided by antibodies to CTLA-4, or PD-1/PD-L1. The inhibitor of apoptosis (IAP) family members cellular (c)-IAP1 and c-IAP2 provide another attractive target for complementary immunotherapy 
through their regulation of both innate and adaptive immune signaling, as well as their ability to modify tumor cell sensitivity to cytokine-mediated cell death [20-24]. Several pharmaceutical companies have developed small molecule IAP antagonists that have potent activity in preclinical models, and are now undergoing early phase clinical testing $[25-27]$.

\section{The IAP family}

The IAPs are a diverse family of regulatory proteins that were first described as caspase inhibitors. The genomes of most mammals encode 7-8 IAP family proteins that are defined by the presence of at least one baculovirus inhibitory repeat (BIR) domain [3]. The first IAPs were identified in baculovirus where they were found to block apoptosis of insect cells [28-30]. These baculovirus IAPs are indeed caspase binders and function to block apoptosis in infected cells [28-30]. X-linked IAP (XIAP) is the only mammalian IAP with substantial caspase-binding activity, but its role in the regulation of apoptosis may be redundant [31-33]. The murine knockout of XIAP shows essentially normal development and no substantial alterations in sensitivity to apoptotic stimuli, potentially indicating compensation of XIAP loss by other IAPs [31]. Similarly, humans with defects in XIAP do not have global defects in apoptosis [34]. The IAPs are nevertheless highly overexpressed in cancer cells, strongly suggesting that this family of proteins provides a survival advantage for tumors, and indeed the IAPs are now appreciated to play broad roles in the regulation of cell signaling and in proliferation [3].

In addition to the family defining BIR domains, four of the IAPs - XIAP, c-IAP1/2 and melanoma (ML)-IAP - have RING finger E3 ligase domains at their c-terminus. These RING-domain containing IAPs appear to exert their cellular functions both through their E3 ubiquitin ligase activity and as adaptor proteins [3].

The c-IAPs are critical regulators of both classical and alternative NF- $\mathrm{BB}$ signaling, functioning downstream of multiple TNF family receptors through their association with TRAF1 or TRAF2, or indirectly with TRAF3 [35,36]. The two c-IAPs are functionally redundant, and reciprocal regulation of the genes in the mouse leads to minimal consequences in single gene knockouts, however c-IAP1 is more broadly expressed than is c-IAP2 [37,38].

Endogenously, the IAPs are antagonized by the mitochondrial protein second mitochondrial activator of caspases (SMAC) which binds to the IAP BIR domain through a tetrapeptide motif that is also present on several other small proteins [3]. SMAC is released from the mitochondria upon activation of intrinsic apoptosis, but may be regulated by other cellular signaling as well. Several small molecule peptide mimetics that incorporate the structural features of the SMAC tetrapeptide have been developed as cancer therapeutics [3,39-42]. These drugs are collectively known as SMAC mimetics, IAP antagonists or IAP inhibitors. Due to functional redundancy within the IAP family, in addition to therapeutic potential, these drugs have served as important research tools for dissecting the cellular functions of the IAPs.

The original goal of using IAP antagonists as cancer therapy was to induce apoptosis in or sensitize cancer cells to apoptosis by relieving caspase inhibition. However, in preclinical testing, these drugs were found to induce cancer cell apoptosis in only a subset of tumors through an unanticipated, c-IAP-dependent mechanism that required autologous production of TNF $\alpha$ [39-42].

The c-IAPs were originally characterized as elements of the TNF receptor (TNFR) complex, though their role within that complex was unknown [39-42]. Through a series of elegant, complementary experiments, several groups found that the c-IAPs interact with the TRAF proteins and serve as ubiquitin ligases within the complex to modulate downstream NF- $\mathrm{KB}$ signaling [39-43]. Binding of c-IAP1 to IAP agonists leads to a conformational shift in the protein that facilitates auto-ubiquitination and targeting to the proteasome for destruction - thus acting through a catalytic mechanism to downregulate c-IAP activity [44].

c-IAPs regulate NF-kB-inducing kinase (NIK) through K-48 ubiquitination, maintaining low levels of the kinase at steady state. In the presence of IAP antagonists, NIK levels rise, leading to phosphorylation and activation of

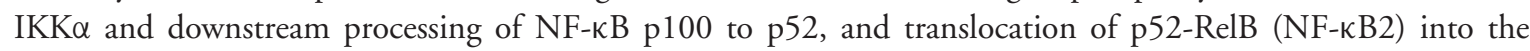
nucleus, thus activating the alternative NF- $\mathrm{BB}$ pathway $[41,42]$. The c-IAPs were also found to regulate classical NF- $\mathrm{BB}$ activation by TNFR1 through K-63 ubiquitination of RIP [45-48]. Ubiquitination of RIP1 has an additional function, blocking its association with a FADD-caspase 8 apoptotic complex that can otherwise assemble at several TNF superfamily receptors, including the TNF $\alpha$ receptor [45-48]. Thus, dual loss of the c-IAPs converts TNFR from a receptor that induces NF- $\mathrm{kB}$ to one that activates caspase-mediated apoptosis upon ligand binding. The few tumor cell lines that are sensitive to this pathway all produced TNF $\alpha$ constitutively, or upon NF- $\kappa$ B2 activation, providing a 'death' ligand through an autocrine loop. Several other cancer cell lines have subsequently been found to be sensitive to IAP antagonists when TNF family receptor ligands such as TRAIL are provided exogenously [20,21]. 


\section{Regulation of TNF $\alpha$ superfamily receptor signaling in immune cells by C-IAP1 \& 2}

A wide body of work now demonstrates a critical role for the c-IAPs in the regulation of NF- $\mathrm{B}$ signaling downstream of multiple TNF superfamily receptors, many of which play important regulatory roles in the immune system [23,35,49-51]. In B cells, the c-IAPs regulate signals through both the B-cell activating factor (BAFF) receptor and CD40, both receptors critical to B-cell function [23,49-50]. Stromal cell production of BAFF is required for normal B-cell homeostasis in vivo, with deficiencies resulting in contraction of the B-cell compartment. BAFF is also required for long-term maintenance of $\mathrm{B}$ cells in culture, particularly in the absence of other stimulation. B-cell survival through BAFF is mediated by activation of NF- $\mathrm{B} 2$, which is in turn controlled by c-IAP ubiquitination of NIK. Inhibition of the c-IAPs, or deficiencies in the TRAF proteins to which they bind, leads to tonic activation of NF- $\mathrm{B} 2$ in B cells and BAFF independence [49]. In vitro, IAP agonists prolong B-cell survival, and in vivo, the B-cell compartment expands [23,49].

CD40 is critical for initiation of B-cell transition into the germinal center reaction and upregulation of activationinduced deaminase, the enzyme responsible for initiating somatic hypermutation and class switch recombination. Regulation of CD40 signaling by the c-IAPs is less well studied, but they appear to function, at least in part, as scaffolds that maintain proper localization of the receptor signaling complex [50]. Loss of the c-IAPs interrupts receptor signaling and inhibits activation of JNK [50,52]. In mice with constitutive c-IAP loss, CD40-dependent germinal center formation is inhibited consistent with a defect in CD40 signaling, though whether the c-IAPs are required for proper antibody formation is unknown [49-50,52]. The decrease in germinal center formation may also be mitigated by tonic activation of NF- $\mathrm{KB} 2$ by the IAP antagonists, which mimics signaling through this pathway that is induced transiently when CD40L binds to CD40. Dendritic cells exposed to IAP antagonists increase expression of MHC class I and class II molecules, upregulate co-stimulatory ligands and produce IL-12 [23]. Whether these effects in dendritic cells are mediated by loss of CD40 associated c-IAPs, or via another TNF family receptor, is unclear [23].

The c-IAPs are further implicated in B-cell malignancies, although their functional role in these tumors is less well understood. c-IAP2 is involved in a recurrent translocation in mucosal-associated lymphoid tissue lymphoma, and has been reported to function as an E3 ligase for BCL10 in lymphocytes, although the physiological importance of this activity is unknown [53]. In multiple myeloma, the c-IAPs are frequently involved in bi-allelic deletions resulting in constitutive alternative NF- $\mathrm{KB} 2$ signaling [54,55].

In $\mathrm{T}$ cells, the c-IAPs function as important negative regulators of costimulation [23,56]. T cells express several TNFR superfamily costimulatory receptors upon activation including OX40, 4-1BB and glucocorticoid-induced TNFR (GITR) [2]. These receptors signal in part through NF-кB2, activation of which is again regulated by c-IAP-mediated ubiquitination [23]. Inhibiting the c-IAPs in both murine and human CD4 and CD8 T cells delivers a costimulatory signal that enhances activation in the presence of TCR stimulation [23]. In vitro, this results in increased cytokine production, proliferation and expression of activation markers in both conventional CD4 and CD8 T cells, as well as lipid-reactive NKT cells [23]. NKT cells also require IAP activity for proper development. XIAP has been directly implicated in NKT cell development in humans, and mice show a defect in NKT cell development when early thymic progenitors are exposed ex vivo to IAP antagonists in fetal thymic organ cultures [34,57]. For each of these effects that have been examined in detail, both signaling through the TCR, and activation of NF-кB2 have been required [23]. These initial findings have been corroborated using $\mathrm{T}$ cells that express a dominant-negative c-IAP1 RING domain mutant [58]. The finding that $\mathrm{T}$ cells from these mutant mice also show enhanced activation directly implicates the ubiquitin ligase activity of the c-IAPs in their co-inhibitory function, independent of their structural role [58]. Although NK cells also appear to be regulated by the c-IAPs, the role of the IAPs in this immune subset has not been thoroughly examined [23,59].

\section{Enhancing innate $\&$ adaptive immunity to cancer using IAP antagonists}

The finding that IAP antagonists deliver co-stimulatory signals in T cells suggests that these compounds could be used to stimulate weak immune responses in vivo, augmenting immunity. Indeed, whereas treatment with the IAP antagonist LBW-242 had minimal effects on T-cell numbers or subset ratios, T cells from LBW-242-treated mice stimulated ex vivo demonstrated enhanced responses [23]. Consistent with enhanced T-cell-mediated immunity, LBW-242 was found to synergize with a vaccine against B16 melanoma, delaying tumor growth and prolonging survival [23]. This activity was correlated with increased numbers of antitumor CD8 T cells, and enhanced antitumor cytokine responses in the tumor-draining lymph node, although a requirement for $\mathrm{T}$ cells in this activity was not formally demonstrated, nor were responses from other immune cell types directly assessed [23]. 
Because IAP antagonists appear to enhance T-cell responses only in the presence of a signal through the TCR, the drugs can be used to boost antigen specific responses. NKT cells are a subset of lipid reactive T cells that express an invariant TCR and have multiple regulatory roles, including anticancer activity in a variety of models. Invariant(i) NKT cells in both mice and humans can be activated by a specific lipid antigen initially isolated from marine sponges called $\alpha$-galactosylceramide ( $\alpha$-galcer). Administration of $\alpha$-galcer to mice leads to broad activation of NKT cells, which has potent antitumor activity in the B16F10 pulmonary metastasis model. Co-administration of IAP antagonists in this setting specifically enhances NKT cell responses, and increases antitumor activity [57].

In addition to activity in vaccination or direct T-cell activation models, IAP antagonists have shown impressive activity in multiple myeloma and glioblastoma when combined with checkpoint inhibitors, and show broad activity when combined with oncolytic viruses [20,22,24]. These findings are of particular importance clinically, given the widespread use of these immune modulating agents.

The SMAC mimetic LCL161 came out of a screen for cancer compounds with antitumor activity in the transgenic $\mathrm{Vk}^{*} \mathrm{Myc}$ mouse model of multiple myeloma, the malignant counterpart to antibody-producing plasma $\mathrm{B}$ cells [22]. LCL161 had single agent efficacy comparable to (or exceeding) other common chemotherapies used for this disease [22]. This was a surprising finding given that co-deletion or mutation of cIAP1/2 (BIRC2 and BIRC3), which lie in tandem at chromosomal position $11 \mathrm{q} 22$, occurs in $15 \%$ of myelomas, leading to constitutively active NF-кB2 [54,55]. Interestingly, unlike the majority of solid cancer cell lines that are sensitized to TNF $\alpha$-induced killing by IAP antagonists, multiple myeloma cell lines were resistant in vitro to the combination of LCL161 and $\mathrm{TNF} \alpha$ [22]. In vivo, the antitumor activity was dependent on cell autonomous production of type I interferons, and was associated with activation of phagocytic myeloid cells [22]. These myeloid cells exerted an immediate antitumor effect through phagocytosis of myeloma cells; the mechanism underlying this activity was not fully explored, but involved a soluble factor [22]. This effect rapidly reduced myeloma cell counts, but durable remissions required activation of adaptive immunity that could be augmented by PD-1 inhibition [22]. Whether the IAP antagonists directly contributed to this adaptive response in this model, or contributed only through enhanced myeloma cell phagocytosis is unclear. PD-L1 expression increased in myeloma cells from IAP-antagonist-treated mice, hinting at a potential direct role for the IAPs in regulating PD-L1 expression, although an indirect role cannot be excluded at this point [22].

Based on these exciting preclinical findings in a well-validated model, the investigators rapidly translated their work into a Phase II clinical trial in relapsed, refractory multiple myeloma [22]. Single agent activity was not seen in this setting, but 5/23 patients who received combination therapy with cyclophosphamide achieved durable responses, some lasting longer than a year [22]. Bone marrow biopsies taken during the trial were consistent with increased tumor cell phagocytosis [22].

In contrast to multiple myeloma, glioblastoma cells are sensitive to IAP antagonist combination with TNF $\alpha$ as well as several other innate immune stimuli [20]. In vivo, IAP antagonist-mediated killing in combination with innate immune stimuli led to long-lasting antitumor protection that corresponded to the generation of robust antitumor T-cell responses [20]. Similarly, established intracranial glioblastoma was responsive to combination therapy with IAP antagonist (in this case LCL161 or birinapant) and checkpoint inhibition (anti-PD1 and/or anti-CTLA-4) [20]. Although none of the agents alone induced durable cures, triple therapy with anti-PD1, anti-CTLA-4 and IAP antagonist led to cures in all animals bearing CT-2A glioblastomas [20].

Effective IAP antagonist combination immunotherapy was associated with an expansion of $\mathrm{CD} 8^{+} \mathrm{T}$ cells and a reduction in Tregs; CD8 T cells were required for this effect as was TNF $\alpha$ produced by these cells [20]. Depletion of CD4 T cells after tumor establishment led to tumor regression potentially through loss of tumor infiltrating Tregs, though this mechanism was not investigated [20]. IAP antagonists alone also increased the number PD- $1^{+}$ CD8 $\mathrm{T}$ cells in the tumor, suggesting a mechanism for synergy with anti-PD-1 antibodies [20]. Surprisingly, tumor regression by IAP antagonist combination immunotherapy was dependent on type I interferon signaling, as the therapeutic benefit was lost in animals given a blocking-antibody to the type I interferon receptor (IFNAR1) [20].

The ability of IAP antagonists to eradicate cancer cells is most often explained by their ability to sensitize tumors to TNF $\alpha$-induced cell death via the RIP1-FADD-caspase 8 complex [3]. What is intriguing from this glioblastoma model, though perhaps not wholly unexpected, is that the source of TNF $\alpha$ necessary for combination therapeutic efficacy was the $\mathrm{CD}^{+}$T-cell rather than cells of the innate immune system.

Taken together, these initial reports using a variety of models strongly suggest that IAP antagonists primarily exert their antitumor activity through modulation of innate and adaptive immune signals. Which of these signals will be most important in patients is presently unknown. Additionally, the ideal combination of IAP proteins to 


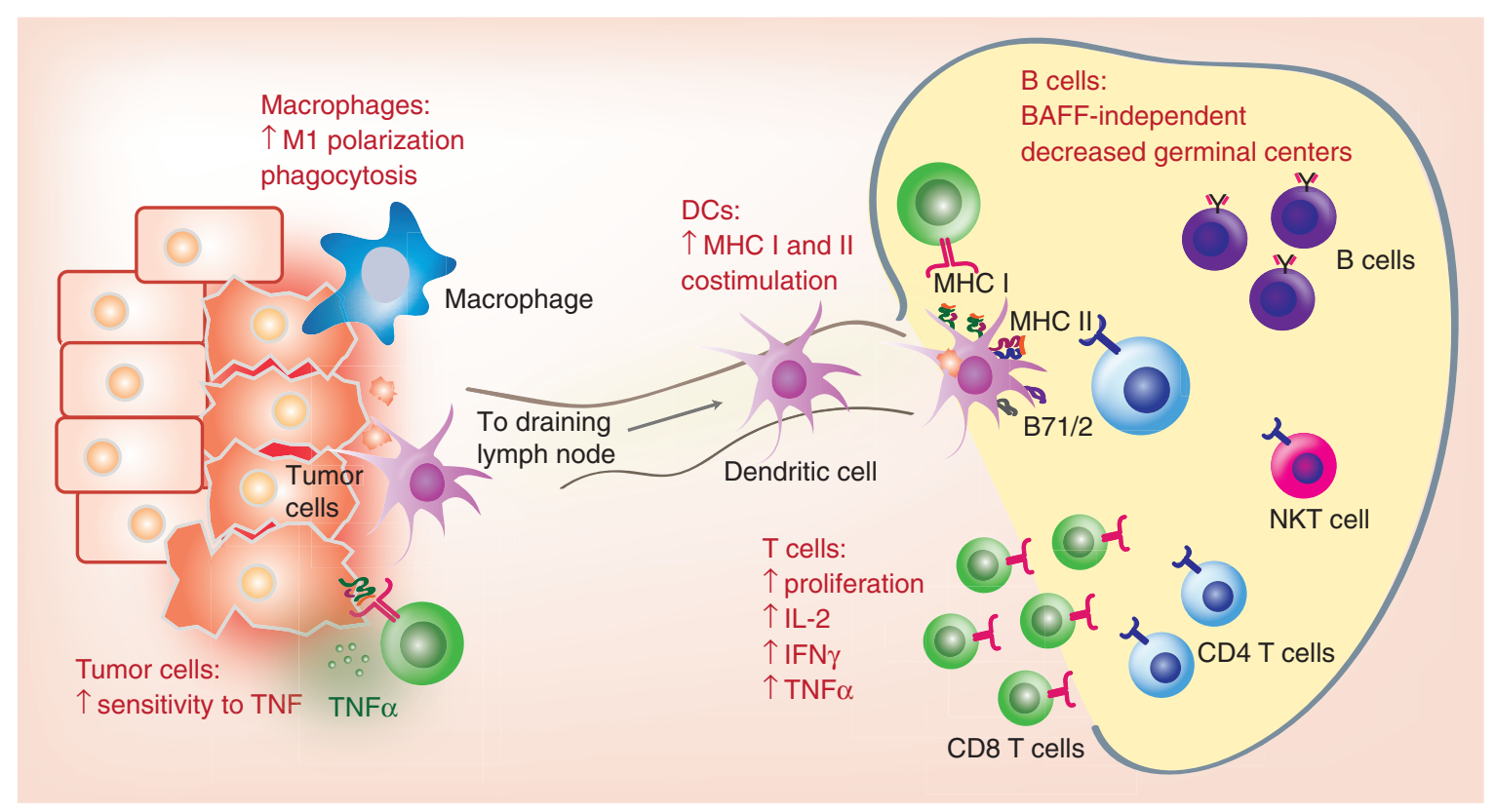

Figure 1. Inhibitor of apoptosis antagonists regulate multiple steps in antitumor immunity.

inhibit in order to leverage this immune modulating effect has not been adequately examined, nor is it clear whether subtle differences in immune activity exist among the currently available pharmacologic inhibitors. Monovalent IAP antagonists such as LCL-161 have preferential activity against the c-IAPs, while bivalent antagonists such as birinapant and BV6 can inhibit and induce degradation of XIAP as well. Whether these differences in activity influence the immune modulating potential of these agents has not been rigorously examined; however, the bivalent antagonists seem to have increased toxicity, consistent with the embryonic lethality seen in animals with combined deficiencies in both c-IAP1 and XIAP [60].

Sensitization to TNF $\alpha$-induced cell death by the IAP antagonists is a feature of many, but not all tumor cell lines examined. Whether this pathway plays an important function in the ability of the IAP antagonists to enhance antitumor responses is an important area for future study, with potential ramifications for selecting other appropriate tumor types for preclinical and clinical testing. Intriguingly, c-IAP1 was a top hit in a screen for tumor intrinsic genes whose loss conferred sensitivity to immune-based therapy [61].

Despite the success of the IAP antagonists in multiple tumor models, both IAP antagonist treatment and expression of the dominant negative cIAP1 RING-mutant inhibited responses to chronic infection in the lymphocytic choriomeningitis virus (LCMV) model [58,62]. This was attributed to a requirement for cIAP1 in 4-1BB signaling, though this hypothesis remains to be fully tested [58,62]. Regardless of the specific mechanism, this finding suggests that in some circumstances, the IAPs can serve in a protective function, and understanding this role may have implications for the use of these drugs as clinical immunotherapeutics.

\section{Future perspective: IAP antagonists as immunotherapy}

Given the myriad regulatory roles for the IAPs in immune cells, systemic treatment with IAP antagonists is likely to have diverse and at times competing effects on evolving immune responses (Figure 1). Multiple distinct IAP antagonists are now in clinical trials. Currently, six trials are ongoing that exploit the immune modulating properties of IAP antagonists, often in combination with other immune therapy (Table 1). In the published Phase I trials, a few substantial clinical responses were seen, though whether the observed activity is immune-mediated remains unknown [25-27]. These drugs have, thus far, been well tolerated, consistent with the general finding that cytotoxicity from these agents typically requires a second signal [25-27].

In animal models, IAP antagonist therapy is considerably more effective when used in combination with other agents, and indeed, durable remissions were only observed in the setting of combination immunotherapy [20,2223]. Although animal models can often underestimate the role of pre-existing immunity in response to immune 
Table 1. Ongoing immunotherapy clinical trials involving IAP antagonist.

\begin{tabular}{|c|c|c|c|c|c|c|}
\hline \multirow[t]{2}{*}{ ClinicalTrials.gov } & \multirow[t]{2}{*}{ Phase } & \multirow[t]{2}{*}{ IAP antagonist } & \multirow[t]{2}{*}{ Valency } & \multicolumn{2}{|c|}{ Combination therapy } & \multirow[t]{2}{*}{ Malignancy } \\
\hline & & & & Immunotherapy & Other agents & \\
\hline NCT01955434 & 2 & LCL-161 & Monovalent & & Cyclophosphamide & $\begin{array}{l}\text { Relapsed or refractory multiple } \\
\text { myeloma }\end{array}$ \\
\hline NCT03111992 & $1 / 1 b$ & LCL-161 & Monovalent & $\begin{array}{l}\text { Anti-IL-17 (CJM112), } \\
\text { anti-PD1 (PDR001) }\end{array}$ & & Multiple myeloma \\
\hline NCT02890069 & $1 b$ & LCL-161 & Monovalent & & $\begin{array}{l}\text { Everolimus (mTOR } \\
\text { inhibitor), panobinostat } \\
\text { (HDAC inhibitor) }\end{array}$ & $\begin{array}{l}\text { Colorectal cancer, non-small-cell lung } \\
\text { carcinoma, triple negative breast cancer }\end{array}$ \\
\hline NCT03270176 & $1 b$ & Debio 1143 & Monovalent & Anti-PD-LI (avelumab) & & $\begin{array}{l}\text { Advanced solid tumors, metastatic } \\
\text { non-small-cell lung carcinoma }\end{array}$ \\
\hline NCT02587962 & $1 / 2$ & Birinapant & Bivalent & $\begin{array}{l}\text { Anti-PD1 } \\
\text { (pembrolizumab) }\end{array}$ & & Refractory solid tumors \\
\hline NCT03166631 & 1 & BI 891065 & Monovalent & Anti-PD1 (BI 754091) & & Advanced/metastatic malignancies \\
\hline
\end{tabular}

modulating treatments, both the co-stimulatory activity of IAP antagonists and the sensitization to TNF superfamily ligand-mediated killing will be most effective when combined with interventions that boost $\mathrm{T}$-cell effector function. These could include vaccines, oncolytic viruses or standard checkpoint blockade as has been reported in mice, but may also extend to other forms of immunotherapy [20,22-24]. With their broad immune stimulatory function, regulating both innate and adaptive immune signals, these drugs promise to become important elements of immunotherapy in the future.

\section{Executive summary}

- Antibodies that target the immunologic 'checkpoints' CTLA-4 and PD-1/PD-L1 have proven effective against diverse human malignancies.

- Yet many human tumors remain resistant, underscoring the need for strategies to expand the reach of immunotherapy.

- The inhibitor of apoptosis (IAP) protein family regulates both innate and adaptive immune signaling, as well as tumor cell sensitivity to cytokine-mediated cell death.

- Small molecular IAP antagonists have potent immune-mediated antitumor immunity in animal models, and represent a potential novel class of immunotherapy.

The inhibitor of apoptosis family

- The IAPs are overexpressed in cancer cells, and cellular (c)-IAP1 and 2 are critical regulators of both classical and alternative NF- $\mathrm{KB}$ signaling downstream of TNF family receptors.

- Endogenously, the IAPs are antagonized by the mitochondrial protein second mitochondrial activator of caspases (SMAC).

- Small molecule SMAC mimetics (also known as IAP antagonists or IAP inhibitors) have been developed as cancer therapeutics.

- IAP antagonists induce cancer cell apoptosis through a c-IAP-dependent mechanism that requires autologous production of TNF $\alpha$.

- The c-IAPs regulate NF-кB-inducing kinase (NIK) through K-48 ubiquitination, maintaining low levels of NIK.

- IAP antagonism increases NIK concentrations, activating IKK $\alpha$ and subsequently NF- $\mathrm{KB} 2$.

- The c-IAPs also ubiquitinate RIP1, blocking its association with the FADD-caspase 8 apoptotic complex; dual loss of the c-IAPs enables association of this complex with TNFR1, converting downstream receptor signaling from NF- $\mathrm{KB}$ induction to caspase activation.

- Consequently, TNFR1 expressing cancer cells that produce TNF $\alpha$ in response to NF-кB2 activation can undergo TNF $\alpha$-mediated apoptosis in response to IAP antagonism.

Regulation of TNF $\alpha$ superfamily receptor signaling in immune cells by c-IAP1 \& 2

- The c-IAPs regulate NF- $\mathrm{B}$ s signaling downstream of multiple TNF superfamily receptors expressed by immune cells.

- In B cells, signals through both the B-cell activating factor (BAFF) receptor and CD40 are regulated by the C-IAPs.

- Activation of BAFF-R signaling by IAP antagonists leads to increased B-cell survival and maintenance in the periphery, while alterations in CD40 signaling reduces germinal center formation.

- Dendritic cells exposed to IAP antagonists increase expression of MHC class I and class II molecules, upregulate co-stimulatory ligands and produce IL-12, although the receptor responsible for this activity is unknown. 
- In T cells, the c-IAPs function as important negative regulators of costimulation.

- T cells express several TNF receptor superfamily costimulatory receptors upon activation including OX40, 4-1BB and glucocorticoid-induced TNF receptor (GITR).

- These receptors signal in part through NF-кB2, activation of which is again regulated by c-IAP-mediated ubiquitination.

- Inhibiting the c-IAPs in both murine and human CD4 and CD8 T cells delivers a costimulatory signal that enhances activation in the presence of TCR stimulation.

- In vitro, this results in increased cytokine production, proliferation and expression of activation markers.

Enhancing innate $\&$ adaptive immunity to cancer using IAP antagonists

- Because of their ability to modulate costimulation, immunotherapy with IAP antagonists has been examined in several animal models.

- IAP antagonists were found to synergize with a vaccine against B16F10 melanoma, delaying tumor growth and prolonging survival.

- This activity correlated with increased antitumor CD8 T cells, and enhanced antitumor cytokine responses.

- Similarly, in the B16F10 model of pulmonary metastasis, activation of invariant (i)NKT cells with $\alpha$-galactosylceramide ( $\alpha$-galcer) synergized with IAP antagonists to clear tumors.

- In addition to activity in vaccination or direct T-cell activation models, IAP antagonists have shown impressive activity in multiple myeloma and glioblastoma when combined with checkpoint inhibitors, and show broad activity when combined with oncolytic viruses.

- Although multiple myeloma cell lines were resistant in vitro to the combination of LCL161 and TNF $\alpha$, in vivo, the antitumor activity was dependent on cell autonomous production of type I interferons, and was associated with activation of phagocytic myeloid cells.

- This effect rapidly reduced myeloma cell counts, but durable remissions required activation of adaptive immunity that could be augmented by PD-1 inhibition.

- Based on these preclinical findings, a Phase II clinical trial was initiated where 5/23 patients who received combination therapy with cyclophosphamide achieved durable responses.

- In contrast to multiple myeloma, glioblastoma cells are sensitive to IAP antagonist when combined with TNF $\alpha$ in vitro.

- In vivo, established intracranial glioblastoma responds to combination therapy with IAP antagonist and checkpoint inhibition.

- Although none of the agents alone induced durable cures, triple therapy with anti-PD1, anti-CTLA-4 and IAP antagonists is curative.

- Tumor regression by IAP antagonist combination immunotherapy is dependent on type I interferon signaling, with the therapeutic benefit abrogated by blocking antibodies to the type I interferon receptor (IFNAR1).

Future perspective: IAP antagonists as immunotherapy

- Multiple IAP antagonists are now in clinical trials. Currently, six trials are ongoing that exploit the immune-modulating properties of IAP antagonists, often in combination with other immune therapies. With their broad immune-stimulatory function, regulating both innate and adaptive immune signals, these drugs promise to become important elements of immunotherapy in the future.

Financial \& competing interests disclosure

SK Dougan reports receiving a commercial research grant from Novartis and is a consultant for the same. SK Dougan received funding from the Dana-Farber Cancer Institute/Novartis Program in Drug Discovery, the Melanoma Research Alliance and the Pew Foundation. M Dougan received funding from the AGA Research Scholars Award, and the National Institute of Diabetes and Digestive and Kidney Diseases (NIDDK) Mentored Clinical Scientist Development Award 1K08DK114563-01. The authors have no other relevant affiliations or financial involvement with any organization or entity with a financial interest in or financial conflict with the subject matter or materials discussed in the manuscript apart from those disclosed.

No writing assistance was utilized in the production of this manuscript.

\section{Open access}

This work is licensed under the Creative Commons Attribution-NonCommercial-NoDerivatives 4.0 Unported License. To view a copy of this license, visit http://creativecommons.org/licenses/by-nc-nd-4.0/ 


\section{References}

Papers of special note have been highlighted as: $\bullet$ of interest

1. Ansell SM, Lesokhin AM, Borrello I et al. PD-1 blockade with nivolumab in relapsed or refractory Hodgkin's lymphoma. N. Engl. J. Med. 372(4), 311-319 (2015).

2. Baumeister SH, Freeman GJ, Dranoff G, Sharpe AH. Coinhibitory pathways in immunotherapy for cancer. Annu. Rev. Immunol. 34, 539-573 (2016).

3. Dynek JN, Vucic D. Antagonists of IAP proteins as cancer therapeutics. Cancer Lett.332(2), 206-214 (2013).

4. Garon EB, Rizvi NA, Hui R et al. Pembrolizumab for the treatment of non-small-cell lung cancer. N. Engl. J. Med. 372(21), 2018-2028 (2015).

5. Hodi FS, O’Day SJ, McDermott DF et al. Improved survival with ipilimumab in patients with metastatic melanoma. N. Engl. J. Med. 363(8), 711-723 (2010).

6. Larkin J, Chiarion-Sileni V, Gonzalez R et al. Combined nivolumab and ipilimumab or monotherapy in untreated melanoma. N. Engl. J. Med. 373(1), 23-34 (2015).

7. Le DT, Uram JN, Wang H et al. PD-1 blockade in tumors with mismatch-repair deficiency. N. Engl. J. Med. 372(26), 2509-2520 (2015).

8. Pardoll DM. The blockade of immune checkpoints in cancer immunotherapy. Nat. Rev. Cancer 12(4), 252-264 (2012).

9. Postow MA, Chesney J, Pavlick AC et al. Nivolumab and ipilimumab versus ipilimumab in untreated melanoma. N. Engl. J. Med. 372(21), 2006-2017 (2015).

10. Robert C, Long GV, Brady B et al. Nivolumab in previously untreated melanoma without BRAF mutation. N. Engl. J. Med. 372(4), 320-330 (2015).

11. Robert C, Thomas L, Bondarenko I et al. Ipilimumab plus dacarbazine for previously untreated metastatic melanoma. N. Engl. J. Med. 364(26), 2517-2526 (2011).

12. Ott PA, Hu Z, Keskin DB et al. An immunogenic personal neoantigen vaccine for patients with melanoma. Nature 547(7662), 217-221 (2017).

13. Rizvi NA, Hellmann MD, Snyder A et al. Cancer immunology. Mutational landscape determines sensitivity to PD-1 blockade in non-small cell lung cancer. Science 348(6230), 124-128 (2015).

14. Schumacher TN, Schreiber RD. Neoantigens in cancer immunotherapy. Science 348(6230), 69-74 (2015).

15. Hugo W, Zaretsky JM, Sun L et al. Genomic and transcriptomic features of response to anti-PD-1 therapy in metastatic melanoma. Cell 165(1), 35-44 (2016).

16. Rooney MS, Shukla SA, Wu CJ, Getz G, Hacohen N. Molecular and genetic properties of tumors associated with local immune cytolytic activity. Cell 160(1-2), 48-61 (2015).

17. Sade-Feldman M, Jiao YJ, Chen JH et al. Resistance to checkpoint blockade therapy through inactivation of antigen presentation. Nat. Commun. 8(1), 1136 (2017).

18. Zaretsky JM, Garcia-Diaz A, Shin DS et al. Mutations associated with acquired resistance to PD-1 blockade in melanoma. N. Engl. J. Med. 375(9), 819-829 (2016).

19. Dougan M, Dranoff G. Immune therapy for cancer. Annu. Rev. Immunol. 27, 83-117 (2009).

20. Beug ST, Beauregard CE, Healy C et al. Smac mimetics synergize with immune checkpoint inhibitors to promote tumour immunity against glioblastoma. Nat. Commun. 8, 14278 (2017).

- First to demonstrate synergy between inhibitor of apoptosis (IAP) antagonists and checkpoint blockade in a solid tumor through an IFN $\alpha$-dependent mechanism.

21. Beug ST, Tang VA, LaCasse EC et al. Smac mimetics and innate immune stimuli synergize to promote tumor death. Nat. Biotechnol. 32(2), 182-190 (2014).

- This paper demonstrates that IAP antagonists can sensitive tumor cells to death induced by inflammatory cytokines such as TNF $\alpha$ and TRAIL, even if those tumor cells are resistant to monotherapy.

22. Chesi M, Mirza NN, Garbitt VM et al. IAP antagonists induce anti-tumor immunity in multiple myeloma. Nat. Med. 22(12), 1411-1420 (2016).

- Shows potent antimyeloma activity for IAP antagonists through induction of tumor cell phagocytosis in an animal, and this antimyeloma response is replicated in a Phase II trial. The authors further show that durable responses in mice are induced by co-treatement with IAP antagonists and anti-PD1 antibodies.

23. Dougan M, Dougan S, Slisz J et al. IAP inhibitors enhance co-stimulation to promote tumor immunity. J. Exp. Med. 207(10), 2195-2206 (2010).

- First to show co-stimulatory activity for IAP antagonists in T cells, and in a melanoma vaccination model, was the first to demonstrate successful immunotherapy using these drugs. 
24. Kim DS, Dastidar H, Zhang C et al. Smac mimetics and oncolytic viruses synergize in driving anticancer T-cell responses through complementary mechanisms. Nat. Commun. 8(1), 344 (2017).

25. Infante JR, Dees EC, Olszanski AJ et al. Phase I dose-escalation study of LCL161, an oral inhibitor of apoptosis proteins inhibitor, in patients with advanced solid tumors. J. Clin. Oncol. 32(28), 3103-3110 (2014).

26. DiPersio JF, Erba HP, Larson RA et al. Oral Debio1143 (AT406), an antagonist of inhibitor of apoptosis proteins, combined with daunorubicin and cytarabine in patients with poor-risk acute myeloid leukemia - results of a Phase I dose-escalation study. Clin. Lymphoma Myeloma Leuk. 15(7), 443-449 (2015).

27. Tolcher AW, Bendell JC, Papadopoulos KP et al. A Phase I dose-escalation study evaluating the safety tolerability and pharmacokinetics of CUDC-427, a potent, oral, monovalent IAP antagonist, in patients with refractory solid tumors. Clin. Cancer Res. 22(18), 4567-4573 (2016).

28. Birnbaum MJ, Clem RJ, Miller LK. An apoptosis-inhibiting gene from a nuclear polyhedrosis virus encoding a polypeptide with Cys/His sequence motifs. J. Virol. 68(4), 2521-2528 (1994).

29. Clem RJ, Miller LK. Control of programmed cell death by the baculovirus genes $p 35$ and iap. Mol. Cell. Biol. 14(8), 5212-5222 (1994).

30. Crook NE, Clem RJ, Miller LK. An apoptosis-inhibiting baculovirus gene with a zinc finger-like motif. J. Virol. 67(4), 2168-2174 (1993).

31. Harlin H, Reffey SB, Duckett CS, Lindsten T, Thompson CB. Characterization of XIAP-deficient mice. Mol. Cell. Biol. 21(10), 3604-3608 (2001).

32. Riedl SJ, Renatus M, Schwarzenbacher R et al. Structural basis for the inhibition of caspase-3 by XIAP. Cell 104(5), 791-800 (2001).

33. Sun C, Cai M, Gunasekera AH et al. NMR structure and mutagenesis of the inhibitor-of-apoptosis protein XIAP. Nature 401(6755), 818-822 (1999).

34. Rigaud S, Fondaneche MC, Lambert $\mathrm{N}$ et al. XIAP deficiency in humans causes an X-linked lymphoproliferative syndrome. Nature 444(7115), 110-114 (2006).

35. Kocab AJ, Veloso A, Paulsen MT, Ljungman M, Duckett CS. Effects of physiological and synthetic IAP antagonism on c-IAP-dependent signaling. Oncogene 34(43), 5472-5481 (2015).

36. Rothe M, Pan MG, Henzel WJ, Ayres TM, Goeddel DV. The TNFR2-TRAF signaling complex contains two novel proteins related to baculoviral inhibitor of apoptosis proteins. Cell 83(7), 1243-1252 (1995).

37. Conte D, Holcik M, Lefebvre CA et al. Inhibitor of apoptosis protein cIAP2 is essential for lipopolysaccharide-induced macrophage survival. Mol. Cell. Biol. 26(2), 699-708 (2006).

38. Conze DB, Albert L, Ferrick DA et al. Posttranscriptional downregulation of c-IAP2 by the ubiquitin protein ligase c-IAP1 in vivo. Mol. Cell. Biol. 25(8), 3348-3356 (2005).

39. Gaither A, Porter D, Yao Y et al. A Smac mimetic rescue screen reveals roles for inhibitor of apoptosis proteins in tumor necrosis factor-alpha signaling. Cancer Res. 67(24), 11493-11498 (2007).

40. Petersen SL, Wang L, Yalcin-Chin A et al. Autocrine TNFalpha signaling renders human cancer cells susceptible to Smac-mimetic-induced apoptosis. Cancer Cell 12(5), 445-456 (2007).

41. Varfolomeev E, Blankenship JW, Wayson SM et al. IAP antagonists induce autoubiquitination of c-IAPs, NF-kappaB activation, and TNFalpha-dependent apoptosis. Cell 131(4), 669-681 (2007).

- Along with Vince et al. in the same issue of Cell, this manuscript demonstrated the mechanism of cIAP regulation of cell death, showing that tumor cells' sensitivity to these drugs depended on autocrine production of TNF $\alpha$ and induction of apoptosis through TNFR1.

42. Vince JE, Wong WW, Khan N et al. IAP antagonists target cIAP1 to induce TNFalpha-dependent apoptosis. Cell 131(4), 682-693 (2007).

- Along with Varfolomeev $e$ al. in the same issue of $\mathrm{Cell}$, this manuscript demonstrated the mechanism of cIAP regulation of cell death, showing that tumor cells' sensitivity to these drugs depended on autocrine production of TNF $\alpha$ and induction of apoptosis through TNFR1.

43. Shu HB, Takeuchi M, Goeddel DV. The tumor necrosis factor receptor 2 signal transducers TRAF2 and c-IAP1 are components of the tumor necrosis factor receptor 1 signaling complex. Proc. Natl Acad. Sci. USA 93(24), 13973-13978 (1996).

44. Dueber EC, Schoeffler AJ, Lingel A et al. Antagonists induce a conformational change in cIAP1 that promotes autoubiquitination. Science 334(6054), 376-380 (2011).

45. Haas TL, Emmerich $\mathrm{CH}$, Gerlach B et al. Recruitment of the linear ubiquitin chain assembly complex stabilizes the TNF-R1 signaling complex and is required for TNF-mediated gene induction. Mol. Cell 36(5), 831-844 (2009).

46. Bertrand MJ, Milutinovic S, Dickson KM et al. cIAP1 and cIAP2 facilitate cancer cell survival by functioning as E3 ligases that promote RIP1 ubiquitination. Mol. Cell 30(6), 689-700 (2008).

47. Mahoney DJ, Cheung HH, Mrad RL et al. Both cIAP1 and cIAP2 regulate TNFalpha-mediated NF-kappaB activation. Proc. Natl Acad. Sci. USA 105(33), 11778-11783 (2008). 
48. Varfolomeev E, Goncharov T, Fedorova AV et al. c-IAP1 and c-IAP2 are critical mediators of tumor necrosis factor alpha (TNFalpha)-induced NF-kappaB activation. J. Biol. Chem. 283(36), 24295-24299 (2008).

49. Zarnegar BJ, Wang Y, Mahoney DJ et al. Noncanonical NF-kappaB activation requires coordinated assembly of a regulatory complex of the adaptors cIAP1, cIAP2, TRAF2 and TRAF3 and the kinase NIK. Nat. Immunol. 9(12), 1371-1378 (2008).

- Found an important role for the cIAPs in B-cell signaling and survival downstream of BAFF through interactions with TRAF2 and TRAF3.

50. Matsuzawa A, Tseng PH, Vallabhapurapu $\mathrm{S}$ et al. Essential cytoplasmic translocation of a cytokine receptor-assembled signaling complex. Science 321(5889), 663-668 (2008).

51. Varfolomeev E, Goncharov T, Maecker H et al. Cellular inhibitors of apoptosis are global regulators of NF-kappaB and MAPK activation by members of the TNF family of receptors. Sci. Signal. 5(216), ra22 (2012).

52. Gardam S, Turner VM, Anderton H et al. Deletion of cIAP1 and cIAP2 in murine B lymphocytes constitutively activates cell survival pathways and inactivates the germinal center response. Blood 117(15), 4041-4051 (2011).

53. Zhou H, Du MQ, Dixit VM. Constitutive NF-kappaB activation by the $\mathrm{t}(11 ; 18)(\mathrm{q} 21 ; \mathrm{q} 21)$ product in MALT lymphoma is linked to deregulated ubiquitin ligase activity. Cancer Cell 7(5), 425-431 (2005).

54. Keats JJ, Fonseca R, Chesi M et al. Promiscuous mutations activate the noncanonical NF-kappaB pathway in multiple myeloma. Cancer Cell 12(2), 131-144 (2007).

55. Annunziata CM, Davis RE, Demchenko Y et al. Frequent engagement of the classical and alternative NF-kappaB pathways by diverse genetic abnormalities in multiple myeloma. Cancer Cell 12(2), 115-130 (2007).

56. Knights AJ, Fucikova J, Pasam A, Koernig S, Cebon J. Inhibitor of apoptosis protein (IAP) antagonists demonstrate divergent immunomodulatory properties in human immune subsets with implications for combination therapy. Cancer Immunol. Immunother. 62(2), 321-335 (2013).

57. Clancy-Thompson E, Ali L, Bruck PT et al. IAP antagonists enhance cytokine production from mouse and human iNKT cells. Cancer Immunol. Res. (2017).

58. Giardino Torchia ML, Munitic I, Castro E, Herz J, McGavern DB, Ashwell JD. c-IAP ubiquitin protein ligase activity is required for 4-1BB signaling and CD8(+) memory T-cell survival. Eur. J. Immunol. 45(9), 2672-2682 (2015).

59. Fischer K, Tognarelli S, Roesler $S$ et al. The Smac mimetic BV6 improves NK cell-mediated killing of rhabdomyosarcoma cells by simultaneously targeting tumor and effector cells. Front. Immunol. 8, 202 (2017).

60. Moulin M, Anderton H, Voss AK et al. IAPs limit activation of RIP kinases by TNF receptor 1 during development. EMBO J. 31(7), 1679-1691 (2012).

61. Manguso RT, Pope HW, Zimmer MD et al. In vivo CRISPR screening identifies Ptpn2 as a cancer immunotherapy target. Nature 547(7664), 413-418 (2017).

62. Gentle IE, Moelter I, Lechler N et al. Inhibitors of apoptosis proteins (IAPs) are required for effective T-cell expansion/survival during antiviral immunity in mice. Blood 123(5), 659-668 (2014). 